\title{
Liouville theorems for stable at infinity solutions of Lane-Emden system
}

\author{
Foued Mtiria ${ }^{\mathrm{a}}$, Dong Ye \\ ${ }^{a}$ ANLIG, UR13ES32, University of Tunis El-Manar, 2092 El Manar II, Tunisia \\ ${ }^{b}$ IECL, UMR 7502, Université de Lorraine, 3 rue Augustin Fresnel, 57073 Metz, France
}

\begin{abstract}
We consider the Lane-Emden system $-\Delta u=v^{p},-\Delta v=u^{\theta}$ in $\mathbb{R}^{N}$, and we prove the nonexistence of smooth positive solutions which are stable outside a compact set, for any $p, \theta>0$ under the Sobolev hyperbola.
\end{abstract}

Keywords: Lane-Emden system, stable solutions, stability outside a compact set, $m$-biharmonic equation.

\section{Introduction}

Consider the classical Lane-Emden system

$$
-\Delta u=v^{p}, \quad-\Delta v=u^{\theta}, \quad u, v>0 \quad \text { in } \mathbb{R}^{N}, \quad \text { where } p, \theta>0 .
$$

There is a famous conjecture who states that: Let $p, \theta>0$. If the pair $(p, \theta)$ is subcritical, i.e. if

$$
\frac{1}{p+1}+\frac{1}{\theta+1}>\frac{N-2}{N}
$$

then there is no smooth solution to 1.1.

The critical curve given by the equality in $(1.2)$ is called the Sobolev hyperbola, which is introduced independently by Mitidieri [12] and Van der Vorst 22, it plays a crucial role in the analysis of (1.1). It is well known that if $(p, \theta)$ lies on or above the Sobolev hyperbola, (1.1) admits radial classical solutions (see [13, 19]), and the Lane-Emden conjecture can be restated as the following: There has no smooth solution to (1.1) if the positive pair $(p, \theta)$ lies below the Sobolev critical hyperbola.

The conjecture is proved to be true for radial functions by Mitidieri [13, Serrin-Zou [18. For the full conjecture, Souto [21, Mitidieri [13] and Serrin-Zou [19] proved that there is no supersolution to [1.1], if $p \theta \leq 1$ or $\max (\alpha, \beta) \geq N-2$, where

$$
\alpha=\frac{2(p+1)}{p \theta-1}, \quad \beta=\frac{2(\theta+1)}{p \theta-1}, \quad p \theta>1 .
$$

Moreover, we can check readily that if $p \theta>1$, the condition 1.2 is equivalent to

$$
N<2+\alpha+\beta .
$$

Therefore, the Lane-Emden conjecture is true in dimensions $N=1,2$. More recently, the conjecture is proved in dimensions $N=3,4$, by Souplet and his collaborators, see [15, 20. For $N \geq 5$, the conjecture is known to be true for $(p, \theta)$ verifying 1.2 and one of the following extra conditions:

- If $p, \theta<\frac{N+2}{N-2}$, see Felmer-de Figuereido [8].

- If $\max (p, \theta) \geq N-3$, see Souplet [20].

Email addresses: mtirifoued@yahoo.fr (Foued Mtiri), dong.ye@univ-lorraine.fr (Dong Ye) 
- If $\min (\alpha, \beta) \geq \frac{N-2}{2}$, see Busca-Manásevich [2]

- If $p=1$ or $\theta=1$, see Lin [11].

These partial results enable us a more restrictive new region for the exponents $(p, \theta)$, which is illustrated by the following figure. In other words, the Lane-Emden conjecture stands open for $N \geq 5, p, \theta>0$ such that

$$
p, \theta \neq 1, \quad \min (\alpha, \beta)<\frac{N-2}{2} \text { and } \max (\alpha, \beta)<N-3 .
$$

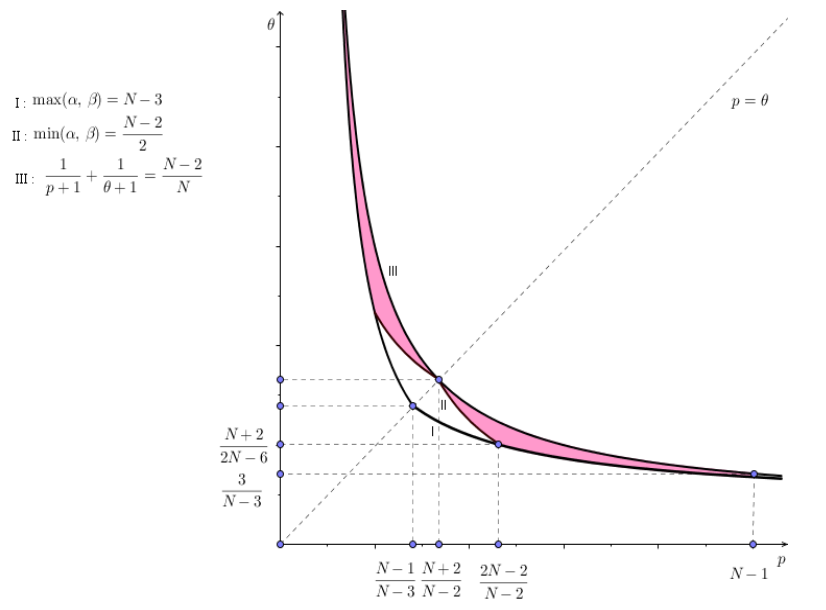

Figure 1: The remained open region (shaded) for the Lane-Emden conjecture $(N \geq 5)$

On the other hand, in the last decade, many efforts were made to obtain some Liouville type result for solutions with finite Morse index, or more generally, which are stable at infinity. To define the notion of stability, we consider a general system given by

$$
-\Delta u=f(x, v), \quad-\Delta v=g(x, u) \text { in } K, \text { a bounded regular domain } \subset \mathbb{R}^{N},
$$

where $f, g \in C^{1}(K \times \mathbb{R})$. Following Montenegro [14, a smooth solution $(u, v)$ of $(1.5)$ is said to be stable in $K$ if the following eigenvalue problem

$$
-\Delta \xi=f_{v}(x, v) \zeta+\eta \xi, \quad-\Delta \zeta=g_{u}(x, u) \xi+\eta \zeta \quad \text { in } K
$$

has a nonnegative eigenvalue $\eta$, with a positive smooth eigenfunctions pair $(\xi, \zeta)$. We say that a pair of solutions $(u, v)$ to $(1.1)$ is stable outside a compact set or stable at infinity, if there is a compact set $K \subset \mathbb{R}^{N}$ such that $(u, v)$ is stable in any bounded domain of $\mathbb{R}^{N} \backslash K$.

For the corresponding second order equation

$$
\Delta u+|u|^{q-1} u=0 \quad \text { in } \mathbb{R}^{N}, \quad q>1,
$$

Farina 7 obtained the optimal Liouville type result for solutions stable at infinity. Indeed, he proved that a smooth nontrivial solution to 1.6 exists, if and only if $q>p_{J L}$ and $N \geq 11$, or $q=\frac{N+2}{N-2}$ and $N \geq 3$. Here $p_{J L}$ denotes the so-called Joseph-Lundgren exponent (see 9, 7). For the biharmornic equation $\Delta^{2} u=|u|^{q-1} u, q>1$, Dávila-Dupaigne-Wang-Wei [5] derived a striking monotonicity formula, which led them to the optimal classification result for solutions stable at infinity, using blow down analysis.

Coming back to the Lane-Emden system (1.1), Chen-Dupaigne-Ghergu [3] studied the stability of radial solutions when $p, \theta \geq 1$. They introduced a new critical hyperbola, called the Joseph-Lundgren

\footnotetext{
${ }^{1}$ In [2], there is another extra condition, which is no longer necessary after the work of Souplet [20].
} 
curve. More precisely, they proved that if $p, \theta \geq 1$, then a radial solution of (1.1) is unstable if and only if $N \leq 10$, or $N \geq 11$ and

$$
\left[\frac{(N-2)^{2}-(\alpha-\beta)^{2}}{4}\right]^{2}<p \theta \alpha \beta(N-2-\alpha)(N-2-\beta) .
$$

Moreover, Cowan proved in [4] that if $p, \theta \geq 2$ and $N \leq 10$, there does not exist any stable solution (radial or not) to (1.1). Recently, Hajlaoui-Harrabi-Mtiri [10] established some Liouville theorems for smooth stable solutions of 1.1 with $p>1$, see Theorem $\mathbf{A}$ below. We mention also the celebrated result of Ramos [17, which states that if $p, \theta>1$ satisfies $(1.2)$, then the system

$$
-\Delta u=|v|^{p-1} v, \quad-\Delta v=|u|^{\theta-1} u \quad \text { in } \mathbb{R}^{N}
$$

does not admit any smooth solutions having finite relative Morse index in the sense of Abbondandolo.

In this paper, our motivation are twofold. We want to obtain the classification results for solutions (radial or not) to 1.1 which are just stable at infinity, and we want to handle the case where $p, \theta$ are allowed to be less than 1. So a natural question is: Can we prove the Lane-Emden conjecture with the extra condition that $(u, v)$ is stable at infinity? The answer is affirmative.

Theorem 1.1. For any $p, \theta>0$ satisfying $(1.2)$, the system (1.1) has no classical solution stable outside a compact set.

If $\theta=p$, using Souplet's comparison result (Lemma 2.7 in [20]), we get $u \equiv v$, so the optimal classification result for solutions stable at infinity was already given by Farina. The classification is also known for $p \theta \leq 1$ as mentioned above. Without loss of generality, we consider only $\theta>p>0$ and $p \theta>1$. As we will see soon, the $\theta>p \geq 1$ case can be handled by the results in [10], so our main concern is the case

$$
\theta p>1>p>0
$$

Let $(u, v)$ be a smooth solution to 1.1 with $\theta>p^{-1}>1>p>0$. Our approach is based on the formal equivalence noticed in [1, 6], between the Lane-Emden system (1.1) and a fourth order problem, called the $m$-biharmonic equation. More precisely, let $m:=\frac{1}{p}+1>2$, as $v=(-\Delta u)^{m-1}$, we derive that $u$ satisfies $\Delta_{m}^{2} u:=\Delta\left(|\Delta u|^{m-2} \Delta u\right)=u^{\theta}$ in $\mathbb{R}^{N}$. So we are led to consider $\theta>m-1>1$ and

$$
\Delta_{m}^{2} u:=\Delta\left(|\Delta u|^{m-2} \Delta u\right)=|u|^{\theta-1} u
$$

Let $\Omega \subset \mathbb{R}^{N}$, we say that $u \in W_{\text {loc }}^{2, m}(\Omega) \cap L_{\text {loc }}^{\theta+1}(\Omega)$ is a weak solutions of 1.7 in $\Omega$, if for any regular bounded domain $K \subset \Omega, u$ is a critical point of the following functional

$$
I(v)=\frac{1}{m} \int_{K}|\Delta v|^{m} d x-\frac{1}{\theta+1} \int_{K}|v|^{\theta+1} d x, \quad \forall v \in W^{2, m}(K) \cap L^{\theta+1}(K) .
$$

Naturally, a weak solution to (1.7) is said stable in $\Omega \subset \mathbb{R}^{N}$, if

$$
\Lambda_{u}(h):=(m-1) \int_{\Omega}|\Delta u|^{m-2}|\Delta h|^{2} d x-\theta \int_{\Omega}|u|^{\theta-1} h^{2} d x \geq 0, \quad \forall h \in C_{c}^{2}(\Omega) .
$$

A key point for our approach is to remark a relationship between the stability for the system (1.1) and the stability for the equation (1.7) (see Lemma 2.1 below). This will permit us to handle the case $0<p<1$ in (1.1) by using the structure of the $m$-biharmonic equation. In fact, we can prove the following Liouville type result.

Theorem 1.2. Let $\theta>m-1>1$ and $u \in W_{l o c}^{2, m}\left(\mathbb{R}^{N}\right) \cap L_{l o c}^{\theta+1}\left(\mathbb{R}^{N}\right)$ be a weak solution of (1.7) which is stable outside a compact set. Assume that

$$
N<\frac{2 m(\theta+1)}{\theta-(m-1)}
$$

then $u \equiv 0$. 
A direct calculation yields that if $p \theta>1$ (or equivalently $\theta>m-1$ ),

$$
N<2+\alpha+\beta=\frac{2(p+1)(\theta+1)}{p \theta-1} \Leftrightarrow 1.9 \Leftrightarrow \theta<\frac{N(m-1)+2 m}{(N-2 m)_{+}} .
$$

It means that the range of pairs $(p, \theta)$ satisfying 1.2$)$ and $p \theta>1$ corresponds exactly to the subcritical case of the $m$-biharmonic equation (1.7).

Another crucial step in our approach is to classify first the stable solutions of (1.1), see also Proposition 2.1 below for the $m$-biharmonic equation.

Proposition 1.1. If $p, \theta>0$ satisfies (1.2), then 1.1) has no smooth stable solution.

Establishing a Liouville type result for stable solution of 1.1 or 1.7 is delicate, even we can borrow some ideas from [23, 5]. We use as usual the stability to get some integral estimates, but the integrations by parts argument yields here many terms which are difficult to control, for example, the local $L^{m}$ norm of $\nabla u$, see Lemma 2.3 below. Furthermore, the classification of weak solutions stable at infinity to (1.7) is more involved than to handle (1.1), since the weak solutions to (1.7) are not $C^{2}$ functions. We will derive a variant of the Pohozaev identity with cut-off functions, which allows us to avoid the spherical integral terms in the standard Pohozaev identity.

The paper is organized as follows. In section 2, we give the proof of Proposition 1.1. The proofs of Theorem 1.1 and Theorem 1.2 are given respectively in sections 3 and 4 . In the following, $C$ denotes always a generic positive constant, which could be changed from one line to another.

\section{Classification of stable solutions}

We prove here Proposition 1.1. As mentioned before, we need only to consider the case $\theta>p$ and $p \theta>1$. We split the proof into two cases: $\theta>p \geq 1$ and $\theta>p^{-1}>1>p>0$.

\subsection{The case $\theta>p \geq 1$.}

Let us recall a consequence of Theorem 1.1 (with $\alpha=0$ there) in [10].

Theorem A. Let $x_{0}$ be the largest root of the polynomial

$$
H(x)=x^{4}-p \theta \alpha \beta\left[4 x^{2}-2(\alpha+\beta) x+1\right] .
$$

(i) If $\frac{4}{3}<p \leq \theta$ then (1.1) has no stable classical solution if $N<2+2 x_{0}$.

(ii) If $1 \leq p \leq \min \left(\frac{4}{3}, \theta\right)$ and $p \theta>1$, then 1.1] has no stable classical solution, if

$$
N<2+2 x_{0}\left[\frac{p}{2}+\frac{(2-p)(p \theta-1)}{(\theta+p-2)(\theta+1)}\right] \text {. }
$$

Performing the change of variables $x=\frac{\beta}{2} s$ in 2.1 , a direct computation shows that $H(x)=\left(\frac{\beta}{2}\right)^{4} L(s)$ where

$$
L(s):=s^{4}-\frac{16 p \theta(p+1)}{\theta+1} s^{2}+\frac{16 p \theta(p+1)(p+\theta+2)}{(\theta+1)^{2}} s-\frac{16 p \theta(p+1)^{2}}{(\theta+1)^{2}} .
$$

Denote by $s_{0}$ the largest root of $L$, hence $x_{0}=\frac{\beta}{2} s_{0}$ and $H(x)<0$ if and only if $L(s)<0$. For $\theta>p \geq 1$, there holds

$$
\begin{aligned}
L(p+1) & =(p+1)^{4}-\frac{16 p \theta(p+1)^{3}}{(\theta+1)}+\frac{16 p \theta(p+1)^{2}(p+\theta+2)}{(\theta+1)^{2}}-\frac{16 p \theta(p+1)^{2}}{(\theta+1)^{2}} \\
& =(p+1)^{4}-\frac{16 p \theta(p+1)^{3}}{(\theta+1)}+\frac{16 p \theta(p+1)^{2}}{(\theta+1)}+\frac{16 p \theta(p+1)^{3}}{(\theta+1)^{2}}-\frac{16 p \theta(p+1)^{2}}{(\theta+1)^{2}} \\
& =(p+1)^{2}\left[(p+1)^{2}-\frac{16 p^{2} \theta}{(\theta+1)}+\frac{16 p^{2} \theta}{(\theta+1)^{2}}\right] \\
& =\left(\frac{p+1}{\theta+1}\right)^{2}\left[(p+1)^{2}(\theta+1)^{2}-16 p^{2} \theta^{2}\right]<0 .
\end{aligned}
$$


The last inequality holds true since

$$
4 p \theta-(p+1)(\theta+1)>4 p^{2}-(p+1)^{2} \geq 0, \quad \forall \theta>p \geq 1 .
$$

As $\lim _{s \rightarrow \infty} L(s)=\infty$, it follows that $s_{0}>p+1$. We get then

$$
2 x_{0}>(p+1) \beta=2+\alpha+\beta, \quad \forall \theta>p \geq 1 .
$$

If $p>\frac{4}{3}$, by ( $i$ ) of Theorem $\mathbf{A}$, the system (1.1) has no classical stable solution if $N<2+\alpha+\beta$. Suppose now $1 \leq p \leq \min \left(\frac{4}{3}, \theta\right)$. Observe that for all $\theta \geq p \geq 1$,

$$
\begin{aligned}
{\left[p+\frac{2(2-p)(p \theta-1)}{(\theta+p-2)(\theta+1)}\right] \beta \geq \alpha+\beta } & \Leftrightarrow\left[p+\frac{2(2-p)(p \theta-1)}{(\theta+p-2)(\theta+1)}\right](\theta+1) \geq p+\theta+2 \\
& \Leftrightarrow p \theta-1+\frac{2(2-p)(p \theta-1)}{\theta+p-2} \geq \theta+1 \\
& \Leftrightarrow(p \theta-1)\left[1+\frac{2(2-p)}{\theta+p-2}\right] \geq \theta+1 \\
& \Leftrightarrow(p \theta-1)(\theta+2-p) \geq(\theta+p-2)(\theta+1) \\
& \Leftrightarrow p \theta^{2}-\theta+(2-p) p \theta \geq \theta^{2}+(p-1) \theta \\
& \Leftrightarrow(p-1)(\theta-p) \geq 0 .
\end{aligned}
$$

As $s_{0}>p+1 \geq 2$, we have $x_{0}=\frac{\beta s_{0}}{2} \geq \beta$ and

$$
2+\alpha+\beta \leq 2+\beta\left[p+\frac{2(2-p)(p \theta-1)}{(\theta+p-2)(\theta+1)}\right] \leq 2+x_{0}\left[p+\frac{2(2-p)(p \theta-1)}{(\theta+p-2)(\theta+1)}\right] .
$$

If $N<2+\alpha+\beta$, using (ii) of Theorem $\mathbf{A}$, we are done.

To conclude, for all $\theta>p \geq 1$ and $N<2+\alpha+\beta$, 1.1) has no smooth stable solution.

\subsection{The case $\theta p>1>p>0$.}

Here we handle the case $0<p<1$. First of all, we need the following lemma which plays an important role in dealing with Proposition 1.1 .

Lemma 2.1. Let $(u, v)$ be a solution of system 1.1) with $\theta>\frac{1}{p}:=m-1>1$. Suppose that $(u, v)$ is stable in a regular bounded domain $\Omega$, then $u$ is a stable solution of equation (1.7).

Proof. By the definition of stability, there exist smooth positive functions $\xi, \zeta$ and $\eta \geq 0$ such that

$$
-\Delta \xi=p v^{p-1} \zeta+\eta \xi, \quad-\Delta \zeta=\theta u^{\theta-1} \xi+\eta \zeta \quad \text { in } \Omega .
$$

Using $(\xi, \zeta)$ as super-solution, $\left(\min _{\bar{\Omega}} \xi, \min _{\bar{\Omega}} \zeta\right)$ as sub-solution, and the standard monotone iterations, we can claim that there exist positive smooth functions $\varphi, \chi$ verifying

$$
-\Delta \varphi=p v^{p-1} \chi, \quad-\Delta \chi=\theta u^{\theta-1} \varphi \quad \text { in } \Omega .
$$

Therefore, we have

$$
\theta u^{\theta-1} \varphi=\Delta\left(\frac{1}{p} v^{1-p} \Delta \varphi\right) \quad \text { in } \Omega
$$

Let $\gamma \in C_{c}^{2}(\Omega)$. Multiplying the above equation by $\gamma^{2} \varphi^{-1}$ and integrating by parts, there holds

$$
\begin{aligned}
\int_{\Omega} \theta u^{\theta-1} \gamma^{2} d x & =\frac{1}{p} \int_{\Omega} v^{1-p} \Delta \varphi \Delta\left(\gamma^{2} \varphi^{-1}\right) d x \\
& =\frac{1}{p} \int_{\Omega} v^{1-p} \Delta \varphi\left(-4 \gamma \frac{\nabla \varphi \cdot \nabla \gamma}{\varphi^{2}}+\frac{2|\nabla \gamma|^{2}}{\varphi}+\frac{2 \gamma \Delta \gamma}{\varphi}+\frac{2 \gamma^{2}|\nabla \varphi|^{2}}{\varphi^{3}}-\frac{\gamma^{2} \Delta \varphi}{\varphi^{2}}\right) d x
\end{aligned}
$$


Using Cauchy-Schwarz's inequality and the fact that $-\Delta \varphi>0$, we get

$$
\left|-4 \int_{\Omega} \frac{v^{1-p}}{p} \Delta \varphi \frac{\nabla \varphi \cdot \nabla \gamma}{\varphi^{2}} \gamma d x\right| \leq-2 \int_{\Omega} \frac{v^{1-p}}{p} \Delta \varphi \frac{|\nabla \gamma|^{2}}{\varphi} d x-2 \int_{\Omega} \frac{v^{1-p}}{p} \Delta \varphi \frac{\gamma^{2}|\nabla \varphi|^{2}}{\varphi^{3}} d x .
$$

Combining (2.2) and (2.3), one obtains, using again the Cauchy-Schwartz inequality,

$$
\begin{aligned}
\int_{\Omega} \theta u^{\theta-1} \gamma^{2} d x & \leq \frac{2}{p} \int_{\Omega} v^{1-p} \Delta \varphi \frac{\gamma \Delta \gamma}{\varphi} d x-\frac{1}{p} \int_{\Omega} v^{1-p} \frac{(\Delta \varphi)^{2}}{\varphi^{2}} \gamma^{2} d x \\
& \leq \frac{1}{p} \int_{\Omega} v^{1-p} \frac{(\Delta \varphi)^{2}}{\varphi^{2}} \gamma^{2} d x+\frac{1}{p} \int_{\Omega} v^{1-p}(\Delta \gamma)^{2} d x-\frac{1}{p} \int_{\Omega} v^{1-p} \frac{(\Delta \varphi)^{2}}{\varphi^{2}} \gamma^{2} d x \\
& =\frac{1}{p} \int_{\Omega} v^{1-p}(\Delta \gamma)^{2} d x .
\end{aligned}
$$

Recall that $p=\frac{1}{m-1}$ and $(-\Delta u)^{\frac{1}{p}}=v$, we obtain the desired result 1.8.

Therefore, to prove Proposition 1.1 in the case $p \in(0,1)$ and $p \theta>1$, we need only to prove

Proposition 2.1. Let $\theta>m-1>1$, if $u$ is a weak stable solution to the equation (1.7) in $\mathbb{R}^{N}$ with $N$ verifying 1.91 , then $u \equiv 0$.

To prove Proposition 2.1 we use first the stability condition $(1.8)$ to get the following crucial lemma which provides an important integral estimate for $u$ and $\Delta u$.

Lemma 2.2. Let $u \in W_{l o c}^{2, m}(\Omega) \cap L_{\text {loc }}^{\theta+1}(\Omega)$ be a weak stable solution of 1.7) in $\Omega$, with $\theta>m-1>1$. Then, for any integer

$$
k \geq \max \left(m, \frac{m(\theta+1)}{2(\theta+1-m)}\right),
$$

there exists a positive constant $C=C(N, \epsilon, m, k)$ such that for any $\zeta \in C_{c}^{2}(\Omega)$ satisfying $0 \leq \zeta \leq 1$,

$$
\int_{\Omega}|\Delta u|^{m} \zeta^{4 k} d x+\int_{\Omega}|u|^{\theta+1} \zeta^{4 k} d x \leq C\left[\int_{\Omega}\left(|\Delta \zeta|^{m}+|\nabla \zeta|^{2 m}+\left|\nabla^{2} \zeta\right|^{m}\right)^{\frac{\theta+1}{\theta-(m-1)}} d x\right] .
$$

Proof. For any $\epsilon \in(0,1)$ and $\eta \in C^{2}(\Omega)$, there holds

$$
\begin{aligned}
\int_{\Omega}|\Delta u|^{m-2}[\Delta(u \eta)]^{2} d x & =\int_{\Omega}|\Delta u|^{m-2}(u \Delta \eta+2 \nabla u \nabla \eta+\eta \Delta u)^{2} d x \\
& \leq(1+\epsilon) \int_{\Omega}|\Delta u|^{m} \eta^{2} d x+\frac{C}{\epsilon} \int_{\Omega}|\Delta u|^{m-2}\left(u^{2}|\Delta \eta|^{2}+|\nabla u|^{2}|\nabla \eta|^{2}\right) d x .
\end{aligned}
$$

Take $\eta=\zeta^{2 k}$ with $\zeta \in C_{c}^{2}(\Omega), 0 \leq \zeta \leq 1$ and $k \geq m>2$. Apply Young's inequality, we get

$$
\begin{aligned}
\int_{\Omega}|u|^{2}|\Delta u|^{m-2}\left|\Delta\left(\zeta^{2 k}\right)\right|^{2} d x & \leq C_{k} \int_{\Omega}|u|^{2}|\Delta u|^{m-2}\left(|\Delta \zeta|^{2}+|\nabla \zeta|^{4}\right) \zeta^{4 k-4} d x \\
& \leq \epsilon^{2} \int_{\Omega}|\Delta u|^{m} \zeta^{4 k} d x+C_{\epsilon, k, m} \int_{\Omega}|u|^{m}\left(|\Delta \zeta|^{2}+|\nabla \zeta|^{4}\right)^{\frac{m}{2}} \zeta^{4 k-2 m} d x
\end{aligned}
$$

and

$$
\begin{aligned}
\int_{\Omega}|\Delta u|^{m-2}|\nabla u|^{2}\left|\nabla\left(\zeta^{2 k}\right)\right|^{2} d x & =4 k^{2} \int_{\Omega}|\Delta u|^{m-2}|\nabla u|^{2}|\nabla \zeta|^{2} \zeta^{4 k-2} d x \\
& \leq \epsilon^{2} \int_{\Omega}|\Delta u|^{m} \zeta^{4 k} d x+\frac{C_{m, k}}{\epsilon^{2}} \int_{\Omega}|\nabla u|^{m}|\nabla \zeta|^{m} \zeta^{4 k-m} d x .
\end{aligned}
$$


Inserting the two above estimates into $(2.5)$, we arrive at

$$
\begin{aligned}
\int_{\Omega}|\Delta u|^{m-2}\left[\Delta\left(u \zeta^{2 k}\right)\right]^{2} d x \leq & (1+C \epsilon) \int_{\Omega}|\Delta u|^{m} \zeta^{4 k} d x+\frac{C_{m, k}}{\epsilon^{3}} \int_{\Omega}|\nabla u|^{m}|\nabla \zeta|^{m} \zeta^{4 k-m} d x \\
& +C_{\epsilon, m, k} \int_{\Omega}|u|^{m}\left(|\Delta \zeta|^{2}+|\nabla \zeta|^{4}\right)^{\frac{m}{2}} \zeta^{4 k-2 m} d x .
\end{aligned}
$$

We need also the following technical lemma, which proof is given later.

Lemma 2.3. Let $k \geq m / 2>1$ and $\epsilon>0$, there exists $C_{N, \epsilon, m, k}>0$ such that for any $u \in W_{l o c}^{2, m}(\Omega)$ verifying 1.8 and $\zeta \in C_{c}^{\infty}(\Omega)$ with $0 \leq \zeta \leq 1$, there holds

$$
\int_{\Omega}|\nabla u|^{m}|\nabla \zeta|^{m} \zeta^{4 k-m} d x \leq \epsilon \int_{\Omega}|\Delta u|^{m} \zeta^{4 k} d x+C_{N, \epsilon, m, k} \int_{\Omega}|u|^{m}\left(|\nabla \zeta|^{2 m}+\left|\nabla^{2} \zeta\right|^{m}\right) \zeta^{4 k-2 m} d x .
$$

Using Lemma 2.3 with $\epsilon^{4}$ and $(2.6)$, we see that

$$
\begin{aligned}
\int_{\Omega}|\Delta u|^{m-2}\left[\Delta\left(u \zeta^{2 k}\right)\right]^{2} d x \leq & C_{N, \epsilon, m, k} \int_{\Omega}|u|^{m}\left(|\Delta \zeta|^{m}+|\nabla \zeta|^{2 m}+\left|\nabla^{2} \zeta\right|^{m}\right) \zeta^{4 k-2 m} d x \\
& +\left(1+C_{m, k} \epsilon\right) \int_{\Omega}|\Delta u|^{m} \zeta^{4 k} d x
\end{aligned}
$$

Thanks to the approximation argument, the stability property (1.8) holds true with $u \zeta^{2 k}$. We deduce then, for any $\epsilon>0$, there exists $C_{N, \epsilon, m, k}>0$ such that

$$
\begin{aligned}
& \theta \int_{\Omega}|u|^{\theta+1} \zeta^{4 k} d x-(m-1)\left(1+C_{m, k} \epsilon\right) \int_{\Omega}|\Delta u|^{m} \zeta^{4 k} d x \\
\leq & C_{N, \epsilon, m, k} \int_{\Omega}|u|^{m}\left(|\Delta \zeta|^{m}+|\nabla \zeta|^{2 m}+\left|\nabla^{2} \zeta\right|^{m}\right) \zeta^{4 k-2 m} d x .
\end{aligned}
$$

Moreover, multiplying the equation (1.7) by $u \zeta^{4 k}$ and integrating by parts, there holds

$$
\int_{\Omega}|\Delta u|^{m} \zeta^{4 k} d x-\int_{\Omega}|u|^{\theta+1} \zeta^{4 k} d x \leq \int_{\Omega}|u||\Delta u|^{m-1}\left|\Delta\left(\zeta^{4 k}\right)\right| d x+C \int_{\Omega}|\Delta u|^{m-1}|\nabla u|\left|\nabla\left(\zeta^{4 k}\right)\right| d x .
$$

Using Young's inequality and applying again Lemma 2.3, we can conclude that for any $\epsilon>0$, there exists $C_{N, \epsilon, m, k}>0$ such that

$$
\begin{aligned}
& \left(1-C_{m, k} \epsilon\right) \int_{\Omega}|\Delta u|^{m} \zeta^{4 k} d x-\int_{\Omega}|u|^{\theta+1} \zeta^{4 k} d x \\
\leq & C_{N, \epsilon, m, k} \int_{\Omega}|u|^{m}\left(|\Delta \zeta|^{m}+|\nabla \zeta|^{2 m}+\left|\nabla^{2} \zeta\right|^{m}\right) \zeta^{4 k-2 m} d x .
\end{aligned}
$$

Taking $\epsilon>0$ but small enough, multiplying 2.10 by $\frac{(m-1)\left(1+2 C_{m, k} \epsilon\right)}{1-C_{m, k} \epsilon}$, adding it with 2.9 , we get

$$
\begin{aligned}
& (m-1) C_{m, k} \epsilon \int_{\Omega}|\Delta u|^{m} \zeta^{4 k} d x+\left[\theta-\frac{(m-1)\left(1+2 C_{m, k} \epsilon\right)}{1-C_{m, k} \epsilon}\right] \int_{\Omega}|u|^{\theta+1} \zeta^{4 k} d x \\
\leq & C_{N, \epsilon, m, k} \int_{\Omega}|u|^{m}\left(|\Delta \zeta|^{m}+|\nabla \zeta|^{2 m}+\left|\nabla^{2} \zeta\right|^{m}\right) \zeta^{4 k-2 m} d x .
\end{aligned}
$$

As $\theta>m-1>1$, using $\epsilon>0$ small enough, we have

$$
\int_{\Omega}|\Delta u|^{m} \zeta^{4 k} d x+\int_{\Omega}|u|^{\theta+1} \zeta^{4 k} d x \leq C \int_{\Omega}|u|^{m}\left(|\Delta \zeta|^{m}+|\nabla \zeta|^{2 m}+\left|\nabla^{2} \zeta\right|^{m}\right) \zeta^{4 k-2 m} d x .
$$


For $k \geq \frac{m(\theta+1)}{2(\theta+1-m)}$ so that $4 k m \leq(4 k-2 m)(\theta+1)$, Applying Hölder inequality, we conclude then

$$
\begin{aligned}
& \int_{\Omega}|\Delta u|^{m} \zeta^{4 k} d x+\int_{\Omega}|u|^{\theta+1} \zeta^{4 k} d x \\
\leq & C\left[\int_{\Omega}\left(|\Delta \zeta|^{m}+|\nabla \zeta|^{2 m}+\left|\nabla^{2} \zeta\right|^{m}\right)^{\frac{\theta+1}{\theta-(m-1)}} d x\right]^{\frac{\theta-(m-1)}{\theta+1}}\left(\int_{\Omega}|u|^{\theta+1} \zeta^{\frac{(4 k-2 m)(\theta+1)}{m}} d x\right)^{\frac{m}{\theta+1}} \\
\leq & C\left[\int_{\Omega}\left(|\Delta \zeta|^{m}+|\nabla \zeta|^{2 m}+\left|\nabla^{2} \zeta\right|^{m}\right)^{\frac{\theta+1}{\theta-(m-1)}} d x\right]^{\frac{\theta-(m-1)}{\theta+1}}\left(\int_{\Omega}|u|^{\theta+1} \zeta^{4 k} d x\right)^{\frac{m}{\theta+1}} .
\end{aligned}
$$

We get readily the estimate (2.4).

Now we choose $\phi_{0}$ a cut-off function in $C_{c}^{\infty}\left(B_{2}\right)$ verifying $0 \leq \phi_{0} \leq 1$ and $\phi_{0}=1$ in $B_{1}$. Applying 2.4 with $\zeta=\phi_{0}\left(R^{-1} x\right)$ for $R>0$, there holds

$$
\int_{B_{R}}|u|^{\theta+1} d x \leq \int_{\mathbb{R}^{N}}|u|^{\theta+1} \zeta^{4 k} d x \leq C R^{\frac{N(\theta-m+1)}{\theta+1}-2 m} .
$$

Under the assumption 1.9 , tending $R \rightarrow \infty$, we obtain $u \equiv 0$, we prove then Proposition 2.1. hence the case $\theta p>1>p>0$ for Proposition 1.1 .

Proof of Lemma 2.3. A direct calculation gives

$$
\begin{aligned}
\int_{\Omega}|\nabla u|^{m}|\nabla \zeta|^{m} \zeta^{4 k-m} d x= & \int_{\Omega} \nabla u \cdot \nabla u|\nabla u|^{m-2}|\nabla \zeta|^{m} \zeta^{4 k-m} d x \\
= & -\int_{\Omega} \operatorname{div}\left(\nabla u|\nabla u|^{m-2}\right) u \mid \nabla \zeta^{m} \zeta^{4 k-m} d x \\
& -\int_{\Omega} u|\nabla u|^{m-2} \nabla u \cdot \nabla\left(|\nabla \zeta|^{m} \zeta^{4 k-m}\right) d x \\
:= & I_{1}+I_{2} .
\end{aligned}
$$

The integral $I_{1}$ can be estimated as

$$
\begin{aligned}
I_{1} & =-(m-2) \int_{\Omega} u|\nabla u|^{m-4}|\nabla \zeta|^{m} \nabla^{2} u(\nabla u, \nabla u) \zeta^{4 k-m} d x-\int_{\Omega} u \Delta u|\nabla u|^{m-2}|\nabla \zeta|^{m} \zeta^{4 k-m} d x \\
& \leq C_{m} \int_{\Omega}|u|\left|\nabla^{2} u\right||\nabla u|^{m-2}|\nabla \zeta|^{m} \zeta^{4 k-m} d x+\int_{\Omega}|u||\Delta u||\nabla u|^{m-2}|\nabla \zeta|^{m} \zeta^{4 k-m} d x .
\end{aligned}
$$

Applying Young's inequality, there holds, for any $\epsilon>0$,

$$
\begin{aligned}
& \int_{\Omega}|u||\Delta u||\nabla u|^{m-2}|\nabla \zeta|^{m} \zeta^{4 k-m} d x \\
\leq & C_{\epsilon, m} \int_{\Omega}|u|^{\frac{m}{2}}|\Delta u|^{\frac{m}{2}}|\nabla \zeta|^{m} \zeta^{4 k-m} d x+\epsilon \int_{\Omega}|\nabla u|^{m}|\nabla \zeta|^{m} \zeta^{4 k-m} d x \\
\leq & C_{\epsilon, m} \int_{\Omega}|u|^{m}|\nabla \zeta|^{2 m} \zeta^{4 k-2 m} d x+\epsilon \int_{\Omega}|\Delta u|^{m} \zeta^{4 k} d x+\epsilon \int_{\Omega}|\nabla u|^{m}|\nabla \zeta|^{m} \zeta^{4 k-m} d x .
\end{aligned}
$$

On the other hand,

$$
\begin{aligned}
& \int_{\Omega}|u|\left|\nabla^{2} u\right||\nabla u|^{m-2}|\nabla \zeta|^{m-2+2} \zeta^{4 k-m} d x \\
\leq & C_{\epsilon, m} \int_{\Omega}|u|^{\frac{m}{2}}\left|\nabla^{2} u\right|^{\frac{m}{2}}|\nabla \zeta|^{m} \zeta^{4 k-m} d x+\epsilon \int_{\Omega}|\nabla u|^{m}|\nabla \zeta|^{m} \zeta^{4 k-m} d x \\
\leq & C_{\epsilon, m} \int_{\Omega}|u|^{m}|\nabla \zeta|^{2 m} \zeta^{4 k-2 m} d x+\epsilon \int_{\Omega}\left|\nabla^{2} u\right|^{m} \zeta^{4 k} d x+\epsilon \int_{\Omega}|\nabla u|^{m}|\nabla \zeta|^{m} \zeta^{4 k-m} d x .
\end{aligned}
$$


Now we shall estimate the integral

$$
\int_{\Omega}\left|\nabla^{2} u\right|^{m} \zeta^{4 k} d x
$$

Remark that there exists $C_{0}(N, m)>0$ such that

$$
\int_{\mathbb{R}^{N}}\left|\nabla^{2} \varphi\right|^{m} d x \leq C_{0}(N, m) \int_{\mathbb{R}^{N}}|\Delta \varphi|^{m} d x, \quad \forall \varphi \in W^{2, m}\left(\mathbb{R}^{N}\right) .
$$

We can prove it firstly for $\varphi \in W_{0}^{2, m}\left(B_{1}\right)$ with elliptic theory, then for general $\varphi \in W^{2, m}\left(\mathbb{R}^{N}\right)$ with approximation and scaling argument. As $u \zeta \in W_{0}^{2, m}(\Omega) \subset W^{2, m}\left(\mathbb{R}^{N}\right)$, 2.15 implies that

$$
\begin{aligned}
\int_{\Omega}\left|\nabla^{2}\left(u \zeta^{\frac{4 k}{m}}\right)\right|^{m} d x \leq & C_{0}(N, m) \int_{\Omega}\left|\Delta\left(u \zeta^{\frac{4 k}{m}}\right)\right|^{m} d x \\
\leq & C_{N, m} \int_{\Omega}|\Delta u|^{m} \zeta^{4 k} d x+C_{N, m, k} \int_{\Omega}|\nabla u|^{m}|\nabla \zeta|^{m} \zeta^{4 k-m} d x \\
& +C_{N, m, k} \int_{\Omega}|u|^{m}\left(|\nabla \zeta|^{2 m}+\left|\nabla^{2} \zeta\right|^{m}\right) \zeta^{4 k-2 m} d x
\end{aligned}
$$

Let $k>m$, we get then

$$
\begin{aligned}
\int_{\Omega}\left|\nabla^{2} u\right|^{m} \zeta^{4 k} d x \leq & C \int_{\Omega}\left|\nabla^{2}\left(u \zeta^{\frac{4 k}{m}}\right)\right|^{m} d x+C_{m, k} \int_{\Omega}|\nabla u|^{m}|\nabla \zeta|^{m} \zeta^{4 k-m} d x \\
& +C_{m, k} \int_{\Omega}|u|^{m}\left(|\nabla \zeta|^{2 m}+\left|\nabla^{2} \zeta\right|^{m}\right) \zeta^{4 k-2 m} d x \\
\leq & C_{N, m} \int_{\Omega}|\Delta u|^{m} \zeta^{4 k} d x+C_{N, m, k} \int_{\Omega}|\nabla u|^{m}|\nabla \zeta|^{m} \zeta^{4 k-m} d x \\
& +C_{N, m, k} \int_{\Omega}|u|^{m}\left(|\nabla \zeta|^{2 m}+\left|\nabla^{2} \zeta\right|^{m}\right) \zeta^{4 k-2 m} d x .
\end{aligned}
$$

Combining (2.13), (2.14) and (2.16), we arrive at

$$
\begin{aligned}
I_{1} \leq & C_{N, m, k} \epsilon \int_{\Omega}|\Delta u|^{m} \zeta^{4 k} d x+C_{m} \epsilon \int_{\Omega}|\nabla u|^{m}|\nabla \zeta|^{m} \zeta^{4 k-m} d x \\
& +C_{N, \epsilon, m, k} \int_{\Omega}|u|^{m}\left(|\nabla \zeta|^{2 m}+\left|\nabla^{2} \zeta\right|^{m}\right) \zeta^{4 k-2 m} d x .
\end{aligned}
$$

Furthermore, by Young's inequality,

$$
\begin{aligned}
I_{2}= & -m \int_{\Omega} u|\nabla u|^{m-2}|\nabla \zeta|^{m-2} \nabla^{2} \zeta(\nabla \zeta, \nabla u) \zeta^{4 k-m} d x \\
& -(4 k-m) \int_{\Omega} u|\nabla u|^{m-2}|\nabla \zeta|^{m}(\nabla u \cdot \nabla \zeta) \zeta^{4 k-m-1} d x \\
\leq & C_{m, k} \int_{\Omega}|u||\nabla u|^{m-1}|\nabla \zeta|^{m-1}\left(|\nabla \zeta|^{2}+\left|\nabla^{2} \zeta\right|\right) \zeta^{4 k-m-1} d x \\
\leq & C_{\epsilon, m, k} \int_{\Omega}|u|^{m}\left(|\nabla \zeta|^{2 m}+\left|\nabla^{2} \zeta\right|^{m}\right) \zeta^{4 k-2 m} d x+\epsilon \int_{\Omega}|\nabla u|^{m}|\nabla \zeta|^{m} \zeta^{4 k-m} d x .
\end{aligned}
$$

Combining (2.17)-2.18) with 2.12, one concludes

$$
\begin{aligned}
\left(1-C_{N, m, k} \epsilon\right) \int_{\Omega}|\nabla u|^{m}|\nabla \zeta|^{m} \zeta^{4 k-m} d x & \leq C_{N, \epsilon, m, k} \int_{\Omega}|u|^{m}\left(|\nabla \zeta|^{2 m}+\left|\nabla^{2} \zeta\right|^{m}\right) \zeta^{4 k-2 m} d x \\
& +C_{N, m, k} \epsilon \int_{\Omega}|\Delta u|^{m} \zeta^{4 k} d x .
\end{aligned}
$$

This means that 2.7 holds true for $\epsilon>0$ small enough, hence for any $\epsilon>0$. 


\section{Proof of Theorem 1.1.}

In this section, we prove Theorem 1.1. As already mentioned, we need only to handle the case $p \theta>1$. We use first the classification for stable solutions, Proposition 1.1 to obtain the decay estimates for stable at infinity solutions of 1.1 .

Lemma 3.1. Let $p, \theta>0$ verify $p \theta>1$ and (1.4). Let $(u, v)$ be a solution of (1.1) which is stable outside a compact set. Then there exists a constant $C$ such that

$$
\sum_{k \leq 2}\left[|x|^{\alpha+k}\left|\nabla^{k} u(x)\right|+|x|^{\beta+k}\left|\nabla^{k} v(x)\right|\right] \leq C, \quad \forall x \in \mathbb{R}^{N} .
$$

Proof. Assume that $(u, v)$ is stable outside $B_{R_{0}}$. Denote

$$
W(x)=\sum_{k \leq 2}\left[\left|\nabla^{k} u(x)\right|^{\frac{1}{\alpha+k}}+\left|\nabla^{k} v(x)\right|^{\frac{1}{\beta+k}}\right] .
$$

Suppose that (3.1) does not hold true. Let $d(x)=\|x\|-R_{0}$, there holds

$$
\sup _{\mathbb{R}^{N} \backslash B_{R_{0}}} W(x) d(x)=\infty,
$$

or equally there exists a sequence $\left(x_{n}\right)$ such that $\left\|x_{n}\right\|>R_{0}$ and $W\left(x_{n}\right) d\left(x_{n}\right)>n$ for $n \geq 1$. Since $(u, v)$ are smooth in $\mathbb{R}^{N}$, then $d\left(x_{n}\right) \rightarrow \infty$. By the doubling lemma [15], there exists another sequence $\left(y_{n}\right)$ such that for any $n \geq 1,\left\|y_{n}\right\|>R_{0}$,

(i) $W\left(y_{n}\right) d\left(y_{n}\right) \geq n$;

(ii) $W\left(y_{n}\right) \geq W\left(x_{n}\right)$;

(iii) $W(z) \leq 2 W\left(y_{n}\right)$ for $|z|>R_{0}$ such that $\left|z-y_{n}\right| \leq \frac{n}{W\left(y_{n}\right)}$.

Let $(u, v)$ be a solution of (1.1), consider the sequence of functions

$$
\widetilde{u}_{n}(x)=\lambda_{n}^{\alpha} u\left(y_{n}+\lambda_{n} x\right), \quad \widetilde{v}_{n}(x)=\lambda_{n}^{\beta} v\left(y_{n}+\lambda_{n} x\right), \quad \text { with } \lambda_{n}=W\left(y_{n}\right)^{-1} .
$$

It's well known that $\left(\widetilde{u}_{n}, \widetilde{v}_{n}\right)$ are a sequence of solutions to 1.1$)$. Moreover,

$$
W_{n}(x):=\sum_{k \leq 2}\left(\left|\nabla^{k} \widetilde{u}_{n}(x)\right|^{\frac{1}{\alpha+k}}+\left|\nabla^{k} \widetilde{v}_{n}(x)\right|^{\frac{1}{\beta+k}}\right)=\lambda_{n} W\left(y_{n}+\lambda_{n} x\right), \quad \forall x \in \mathbb{R}^{N} .
$$

By $(i)$, we have $B_{n \lambda_{n}}\left(y_{n}\right) \subset \mathbb{R}^{N} \backslash B_{R_{0}}$, and we can readily check that $\left(\widetilde{u}_{n}, \widetilde{v}_{n}\right)$ is stable in $B_{n}$ since $(u, v)$ is stable in $\mathbb{R}^{N} \backslash B_{R_{0}}$. Using (iii), there holds, for all $n \geq 1$,

$$
W_{n}(x) \leq 2 W_{n}(0)=2 \text { in } B_{n} .
$$

From 3.2 and standard elliptic theory, up to a subsequence, $\left(\widetilde{u}_{n}, \widetilde{v}_{n}\right)$ converges to $\left(u_{\infty}, v_{\infty}\right)$ in $C_{l o c}^{2}\left(\mathbb{R}^{N}\right)$. Therefore

$$
\sum_{k \leq 2}\left(\left|\nabla^{k} u_{\infty}(0)\right|^{\frac{1}{\alpha+k}}+\left|\nabla^{k} v_{\infty}(0)\right|^{\frac{1}{\beta+k}}\right)=1 .
$$

So $\left(u_{\infty}, v_{\infty}\right)$ is nontrivial. Clearly, $\left(u_{\infty}, v_{\infty}\right)$ a smooth positive solution to (1.1). Using again the elliptic theory, it's not difficult to see that $\left(u_{\infty}, v_{\infty}\right)$ is stable in $\mathbb{R}^{N}$. However, this contradicts Proposition 1.1 since $p, \theta$ verifies $(1.2)$. Hence the hypothesis was wrong, i.e. the estimate 3.1 holds true.

Another tool is the following classical Pohozaev identity (see [12, 16, 20]).

Lemma 3.2. Let $(u, v)$ be a solution to (1.1). Therefore for any regular bounded domain $\Omega$,

$$
\begin{aligned}
& \frac{2(p+1)-p N}{p+1} \int_{\Omega} v^{p+1} d x+\frac{N}{\theta+1} \int_{\varnothing} u^{\theta+1} d x \\
= & \int_{\partial \Omega} u^{\theta+1}(\nu \cdot x) d \sigma-\frac{p}{p+1} \int_{\partial \Omega} v^{p+1}(\nu \cdot x) d \sigma+\int_{\partial \Omega} \frac{\partial v}{\partial \nu}(\nabla u \cdot x) d \sigma-\int_{\partial \Omega} v \frac{\partial(\nabla u \cdot x)}{\partial \nu} d \sigma .
\end{aligned}
$$


We claim then

Lemma 3.3. Let $p, \theta>0$ satisfy $p \theta>1$ and (1.4). If $(u, v)$ be a solution of (1.1) which is stable outside a compact set, then $v \in L^{p+1}\left(\mathbb{R}^{N}\right), u \in L^{\theta+1}\left(\mathbb{R}^{N}\right)$ and

$$
\frac{2(p+1)-p N}{p+1} \int_{\mathbb{R}^{N}} v^{p+1} d x+\frac{N}{\theta+1} \int_{\mathbb{R}^{N}} u^{\theta+1} d x=0 .
$$

Proof . By 3.1), we have (noticing that $\alpha(\theta+1)=(p+1) \beta=2+\alpha+\beta)$

$$
u^{\theta+1}(x)+v^{p+1}(x) \leq C(1+|x|)^{-(2+\alpha+\beta)} \quad \text { in } \mathbb{R}^{N} .
$$

By 1.4, then $v \in L^{p+1}\left(\mathbb{R}^{N}\right), u \in L^{\theta+1}\left(\mathbb{R}^{N}\right)$. Using Lemma 3.2 with $\Omega=B_{R}$, we deduce that

$$
\begin{aligned}
& \frac{2(p+1)-p N}{p+1} \int_{B_{R}} v^{p+1} d x+\frac{N}{\theta+1} \int_{B_{R}}|u|^{\theta+1} d x \\
= & \int_{\partial B_{R}}\left[R \frac{\partial u}{\partial r} \frac{\partial v}{\partial r}-\frac{p R}{p+1} v^{p+1}-v \frac{\partial(\nabla u \cdot x)}{\partial r}+\frac{R}{\theta+1}|u|^{\theta+1}\right] d \sigma .
\end{aligned}
$$

Using again (3.1) and $N<2+\alpha+\beta$, we deduce that

$$
\int_{\partial B_{R}}\left[R \frac{\partial u}{\partial r} \frac{\partial v}{\partial r}-\frac{R}{p+1} v^{p+1}-v \frac{\partial(\nabla u \cdot x)}{\partial r}+\frac{R}{\theta+1}|u|^{\theta+1}\right] d \sigma \rightarrow 0, \quad \text { as } \quad R \rightarrow \infty .
$$

Taking the limit $R \rightarrow \infty$ in $(3.5)$, the claim follows.

Proof of Theorem 1.1 completed. We are now in position to conclude. Suppose that $(u, v)$ is a solution to (1.1) stable at infinity with $p \theta>1$ verifying (1.4). Choose $\phi_{0}$ a cut-off function in $C_{c}^{\infty}\left(B_{2}\right)$ verifying $0 \leq \phi_{0} \leq 1$ and $\phi_{0}=1$ in $B_{1}$. Denote $\zeta=\phi_{0}\left(R^{-1} x\right)$ and $A_{R}=B_{2 R} \backslash B_{R}$. By the system (1.1), there holds

$$
\begin{aligned}
\int_{B_{2 R}} v^{p+1} \zeta d x-\int_{B_{2 R}} u^{\theta+1} \zeta d x & =\int_{B_{2 R}} u \zeta \Delta v d x-\int_{B_{2 R}} u \zeta \Delta v d x \\
& =\int_{B_{2 R}} v(2 \nabla u \cdot \nabla \zeta+u \Delta \zeta) d x \\
& \leq \frac{C}{R^{2}} \int_{A_{R}} u v d x+\frac{C}{R} \int_{A_{R}} v|\nabla u| d x
\end{aligned}
$$

Using (3.1), and tending $R \rightarrow \infty$, as $N<2+\alpha+\beta$, we have

$$
\int_{\mathbb{R}^{N}} v^{p+1} d x=\int_{\mathbb{R}^{N}} u^{\theta+1} d x
$$

Substituting this in 3.4 ,

$$
\left(\frac{2(p+1)-p N}{p+1}+\frac{N}{\theta+1}\right) \int_{\mathbb{R}^{N}} u^{\theta+1} d x=0
$$

As (1.4) implies that

$$
\frac{2(p+1)-p N}{p+1}+\frac{N}{\theta+1}=2-\frac{(p \theta-1) N}{(p+1)(\theta+1)}=2-\frac{2 N}{2+\alpha+\beta}>0,
$$

$u \equiv 0$ in $\mathbb{R}^{N}$ which is absurd, so we are done. 


\section{Proof of Theorem 1.2,}

The approach is similar to that for Theorem 1.1. We derive first some integral estimates thanks to Lemma 2.2. Suppose that $u$ is stable outside the ball $B_{R_{0}}$. Let $R>R_{0}+3$ and $\zeta \in C_{c}^{2}\left(\mathbb{R}^{N} \backslash B_{R_{0}}\right)$ verifying that $0 \leq \zeta \leq 1$ and

$$
\zeta(x)= \begin{cases}0 & \text { for }\|x\| \leq R_{0}+1,\|x\| \geq 2 R \\ 1 & \text { for } R_{0}+2 \leq\|x\| \leq R\end{cases}
$$

Clearly, we can assume that there exists $C>0$ independent on $R$ such that

$$
\|\zeta\|_{C^{2}\left(B_{R_{0}+2}\right)} \leq C \quad \text { and } \quad R|\nabla \zeta(x)|+R^{2}\left|\nabla^{2} \zeta(x)\right| \leq C \text { in } A_{R}=B_{2 R} \backslash B_{R} .
$$

Applying the estimate 2.4 with $\zeta$, we get readily

$$
\int_{R_{0}+2 \leq\|x\| \leq R}|\Delta u|^{m} d x+\int_{R_{0}+2 \leq\|x\| \leq R}|u|^{\theta+1} d x \leq C\left(1+R^{N-\frac{2 m(\theta+1)}{\theta-(m-1)}}\right) .
$$

Using $(1.9)$ and tending $R \rightarrow \infty$, we have then

$$
u \in L^{\theta+1}\left(\mathbb{R}^{N}\right) \text { and } \quad \Delta u \in L^{m}\left(\mathbb{R}^{N}\right) .
$$

By Hölder's inequality, there holds

$$
R^{-2 m} \int_{B_{R}}|u|^{m} d x \leq C R^{\frac{N(\theta+1-m)}{\theta+1}-2 m}\left(\int_{B_{R}}|u|^{\theta+1} d x\right)^{\frac{m}{\theta+1}} .
$$

On the other hand, by standard scaling argument, there exists $C>0$ such that for any $R>0$, any $u \in W^{2, m}\left(A_{R}\right)$ with $A_{R}=B_{2 R} \backslash B_{R}$,

$$
R^{-m} \int_{A_{R}}|\nabla u|^{m} d x \leq C \int_{A_{R}}|\Delta u|^{m} d x+C R^{-2 m} \int_{A_{R}}|u|^{m} d x .
$$

Therefore, under the assumptions of Theorem 1.2 , we get

$$
R^{-2 m} \int_{A_{R}}|u|^{m} d x+R^{-m} \int_{A_{R}}|\nabla u|^{m} d x \rightarrow 0 \quad \text { as } R \rightarrow \infty .
$$

Let $\zeta(x)=\phi_{0}\left(R^{-1} x\right)$ with a standard cut-off function $\phi_{0} \in C_{c}^{2}\left(B_{2}\right), \phi_{0} \equiv 1$ in $B_{1}$. Applying the estimate 2.16 and using 4.2 4.3 , there holds

$$
\int_{R^{N}}\left|\nabla^{2} u\right|^{m} d x<\infty
$$

However, as we have mentioned, the weak solutions of 1.7 are in general not belongs to $C^{2}$, so we cannot use the standard Pohozaev identity similar to 3.3 because of the boundary terms. We show here a variant of the Pohozaev identity, which proof is given in the appendix for the convenience of the readers.

Lemma 4.1. Let $u$ be a weak solution to 1.7) with $m>2$. Then for any $\psi \in C_{c}^{2}(\Omega)$,

$$
\begin{aligned}
& \frac{N}{\theta+1} \int_{\Omega}|u|^{\theta+1} \psi d x-\frac{N-2 m}{m} \int_{\Omega}|\Delta u|^{m} \psi d x \\
= & -\frac{1}{\theta+1} \int_{\Omega}|u|^{\theta+1}(\nabla \psi \cdot x) d x+\frac{1}{m} \int_{\Omega}(\nabla \psi \cdot x)|\Delta u|^{m} d x \\
& -\int_{\Omega}|\Delta u|^{m-2}\left[2 \Delta u(\nabla u \cdot \nabla \psi)+2 \Delta u \nabla^{2}(x \cdot \nabla \psi)+\Delta u(\nabla u \cdot x) \Delta \psi\right] d x .
\end{aligned}
$$

This implies that if $\mathrm{u}$ is a weak solution of 1.7 , stable at infinity with $1<m-1<\theta$ and $N$ verifying 
(1.9), then

$$
\frac{N-2 m}{m} \int_{\mathbb{R}^{N}}|\Delta u|^{m} d x=\frac{N}{\theta+1} \int_{\mathbb{R}^{N}}|u|^{\theta+1} d x .
$$

Indeed, let $\psi$ in 4.5 be defined by $\psi(x)=\phi_{0}\left(R^{-1} x\right)$ with a standard cut-off function $\phi_{0} \in C_{c}^{2}\left(B_{2}\right)$, $\phi_{0} \equiv 1$ in $B_{1}$. Denote the right hand side in (4.5) by $I_{R}$. Remark that $\nabla \psi \neq 0$ only in $A_{R}=B_{2 R} \backslash B_{R}$ and $\left\|\nabla^{k} \psi\right\|_{\infty} \leq C_{k} R^{-k}$, we obtain readily

$$
\left|I_{R}\right| \leq C \int_{A_{R}}\left(|u|^{\theta+1}+|\Delta u|^{m}\right) d x+\frac{C}{R} \int_{A_{R}}|\Delta u|^{m-1}|\nabla u| d x+C \int_{A_{R}}|\Delta u|^{m-1}\left|\nabla^{2} u\right| d x
$$

Thanks to the estimates (4.2)-(4.4) and Hölder's inequality, clearly $\lim _{R \rightarrow \infty} I_{R}=0$, hence we get 4.6.

On the other hand, using $u \psi$ as test function in 1.7 , we get

$$
\begin{aligned}
\int_{B_{2 R}}|\Delta u|^{m} \psi d x-\int_{B_{2 R}}|u|^{\theta+1} \psi d x & \leq C \int_{B_{2 R}}|u||\Delta u|^{m-1}|\Delta \psi| d x+C \int_{B_{2 R}}|\Delta u|^{m-1}|\nabla u||\nabla \psi| d x \\
& \leq \frac{C}{R^{2}} \int_{A_{R}}|u||\Delta u|^{m-1} d x+\frac{C}{R} \int_{A_{R}}|\Delta u|^{m-1}|\nabla u| d x .
\end{aligned}
$$

Apply Hölder's inequality, 4.2 4.3 and tending $R$ to $\infty$, we obtain

$$
\int_{\mathbb{R}^{N}}|u|^{\theta+1} d x=\int_{\mathbb{R}^{N}}|\Delta u|^{m} d x
$$

Combining (4.6) and 4.7), one obtains

$$
\left(\frac{N-2 m}{m}-\frac{N}{\theta+1}\right) \int_{\mathbb{R}^{N}}|u|^{\theta+1} d x=0 .
$$

We are done, since 1.9 implies that $\frac{N-2 m}{m}-\frac{N}{\theta+1}<0$.

\section{Appendix}

We prove here the Lemma 4.1. Let $\psi \in C_{c}^{2}(\Omega)$, multiplying equation 1.7 by $\nabla u \cdot x \psi$ and integrating by parts, we get

$$
\begin{aligned}
& \int_{\Omega}|u|^{\theta-1} u(\nabla u \cdot x) \psi d x \\
= & \int_{\Omega}|\Delta u|^{m-2} \Delta u \Delta(\nabla u \cdot x \psi) d x \\
= & \int_{\Omega}|\Delta u|^{m-2} \Delta u[(\nabla(\Delta u) \cdot x) \psi+2 \Delta u \psi+2 \nabla(\nabla u \cdot x) \cdot \nabla \psi+(\nabla u \cdot x) \Delta \psi] d x .
\end{aligned}
$$

Direct calculation yields $\nabla(\nabla u \cdot x) \cdot \nabla \psi=\nabla^{2} u(x, \nabla \psi)+(\nabla u \cdot \nabla \psi)$ and

$$
\begin{aligned}
\int_{\Omega}|\Delta u|^{m-2} \Delta u[(\nabla(\Delta u) \cdot x) \psi+2 \Delta u \psi] d x & =\int_{\Omega} \frac{\nabla|\Delta u|^{m}}{m} \cdot x \psi d x+2 \int_{\Omega}|\Delta u|^{m} \psi d x \\
& =\frac{2 m-N}{m} \int_{\Omega}|\Delta u|^{m} \psi d x-\frac{1}{m} \int_{\Omega}|\Delta u|^{m}(\nabla \psi \cdot x) d x .
\end{aligned}
$$

Moreover,

$$
\begin{aligned}
\int_{\Omega}|u|^{\theta-1} u(\nabla u \cdot x) \psi d x & =-\frac{1}{\theta+1} \int_{\Omega}|u|^{\theta+1} \operatorname{div}(\psi x) d x \\
& =-\frac{N}{\theta+1} \int_{\Omega}|u|^{\theta+1} \psi d x-\frac{1}{\theta+1} \int_{\Omega}|u|^{\theta+1} x \cdot \nabla \psi d x .
\end{aligned}
$$


Therefore, the claim follows by regrouping the above equalities.

\section{Acknowledgment}

The authors are partly supported by the CNRS-DGRST Project No. EDC26348.

\section{References}

\section{References}

[1] D. Bonheure, E.M. Dos Santos and M. Ramos. Symmetry and symmetry breaking for ground state solutions of some strongly coupled elliptic systems. J. Funct. Anal., 264 (2013), 62-96.

[2] J. Busca and R. Manásevich, A Liouville-type theorem for Lane-Emden system, Indiana Univ. Math. J., 51 (2002), 37-51.

[3] W. Chen, L. Dupaigne and M. Ghergu, A new critical curve for the Lane-Emden system, Discrete Contin. Dyn. Syst., 34 (2014), 2469-2479.

[4] C. Cowan, Liouville theorems for stable lane emden systems and biharmonic problems. Nonlinearity, 26 (2013), 2357-2371.

[5] J. Dávila, L. Dupaigne, K. Wang and J. Wei, A monotonicity formula and a Liouville-type theorem for a fourth order supercritical problem, Adv. Math., 258 (2014), 240-285.

[6] E.M. Dos Santos, Multiplicity of solutions for a fourth-order quasilinear nonhomogeneous equation. J. Math. Anal. Appl., 342 (2008), 277-297.

[7] A. Farina, On the classification of solutions of the Lane-Emden equation on unbounded domains of $\mathbb{R}^{N}$, J. Math. Pures Appl., 87 (2007), 537-561.

[8] P. Felmer and D.G. de Figueiredo, A Liouville-type theorem for systems, Ann. Sc. Norm. Sup. Pisa, XXI (1994), 387-397.

[9] C. Gui, W.M. Ni and X.F. Wang, On the stability and instability of positive steady states of a semilinear heat equation in $\mathbb{R}^{N}$, Comm. Pure Appl. Math., 45 (1992), 1153-1181.

[10] H. Hajlaoui, A. Harrabi and F. Mtiri, Liouville theorems for stable solutions of the weighted LaneEmden system, Discrete Contin. Dyn. Syst., 37 (2017), 265-279.

[11] C.S. Lin, A classification of solutions of a conformally invariant fourth order equation in $\mathbb{R}^{n}$, Comment. Math. Helv., 73 (1998), 206-231.

[12] E. Mitidieri, A Rellich type identity and applications, Comm. Partial Diff. Equations, 18 (1993), 125-151.

[13] E. Mitidieri, Nonexistence of positive solutions of semilinear elliptic systems in RN, Diff. Inte. Equations, 9 (1996), 465-479.

[14] M. Montenegro, Minimal solutions for a class of elliptic systems. Bull. London Math. Soc., 37 (2005), 405-416.

[15] P. Poláčik, P. Quittner and P. Souplet, Singularity and decay estimates in superlinear problems via Liouville-type theorems. I. Elliptic equations and systems, Duke Math. J., 139 (2007), 555-579.

[16] P. Pucci and J. Serrin, A general variational identity, Indiana Univ. Math. J., 35 (1986), 681-703.

[17] M. Ramos, A priori bounds via the relative Morse index of solutions of an elliptic system. Topol. Methods Nonlinear Anal., 34 (2009), 21-39. 
[18] J. Serrin, H. Zou, Non-existence of positive solutions of semilinear elliptic systems, Discourses Math. Appl., 3 (1994), 55-68.

[19] J. Serrin and H. Zou, Existence of positive solutions of the Lane-Emden system, Atti Semin. Mat. Fis. Univ. Modena, 46 (1998), 369-380.

[20] P. Souplet, The proof of the Lane-Emden conjecture in four space dimensions, Adv. Math., 221 (2009), 1409-1427.

[21] M.A.S. Souto, A priori estimates and existence of positive solutions of non-linear cooperative elliptic systems, Diff. Inte. Equations, 8 (1995), 1245-1258.

[22] R.C.A.M. Van der Vorst, Variational identities and applications to differ- ential systems, Arch. Rational Mech. Anal., 116 (1992), 375-398.

[23] J. Wei and D. Ye, Liouville theorems for stable solutions of biharmonic problem, Math. Annalen, 356 (2013), 1599-1612. 